\title{
How data governance technologies can democratize data sharing for community well-being - Corrigendum
}

\author{
Dan $\mathrm{Wu}^{1}$, Stefaan G. Verhulst ${ }^{2}$, Alex Pentland ${ }^{3}$, Thiago Avila ${ }^{4}$, Kelsey Finch ${ }^{5}$ and Abhishek Gupta ${ }^{6}$ \\ ${ }^{1}$ Immuta Inc., College Park, Maryland, USA \\ ${ }^{2}$ The GovLab, New York University, New York, New York, USA \\ ${ }^{3}$ MIT Media Lab, Massachusetts Institute of Technology, Cambridge, Massachusetts, USA \\ ${ }^{4}$ Faculdade Estácio de Alagoas, Maceió, Brazil \\ ${ }^{5}$ Future of Privacy Forum, Washington, District of Columbia, USA \\ ${ }^{6}$ Montreal AI Ethics Institute, Montreal, Quebec, Canada
}

https://doi.org/10.1017/dap.2021.13, Published by Cambridge University Press, 13 July 2021.

Key words: corrigendum; data governance technologies; data strategy

This commentary in Data \& Policy (Wu et al., 2021) did not contain affiliation details of all the authors of the article. This correction notice has been issued to update the scholarly record.

The funding statement was also unintentionally excluded from the published article. To clarify: "This work received no specific grant from any funding agency, commercial, or not-for-profit sectors."

\section{Reference}

Wu D, Verhulst S, Pentland A, Avila T, Finch K and Gupta A (2021) How data governance technologies can democratize data sharing for community well-being. Data \& Policy 3, E14. https://doi.org/10.1017/dap.2021.13

Cite this article: Wu D, Verhulst S. G, Pentland A, Avila T, Finch K and Gupta A (2021). How data governance technologies can democratize data sharing for community well-being - Corrigendum. Data \& Policy, 3: e23. doi:10.1017/dap.2021.22 